\title{
Localization of plastic deformation in stretching plates: effect of crystalline structure
}

\author{
Jean-Lin Dequiedt ${ }^{1,2^{*}}$ and Christophe Denoual ${ }^{1,2}$ \\ ${ }^{1}$ CEA, DAM, DIF, F-91297 Arpajon, France \\ ${ }^{2}$ Université Paris-Saclay, CEA, LMCE, F-91680 Bruyères-le-Chatel, France
}

\begin{abstract}
The integration of the polycrystalline structure in the simulation of stretching plates, performed by the random generation of a grain aggregate and a set of lattice orientations, gives new insights into the phenomenon of plastic strain localization in the form of necking, albeit well predicted at the scale of continuum by instability analysis. A transition is displayed from initial grain scale heterogeneity, with some connection to crystal lattice orientation towards stress axes, to the onset of macroscopic localization patterns. Depending on the number of grains and on the stretching rate, it seems that a competition emerges between a "weakest link" process for which the final necks are located in places where initial deformation is high and an instable mode controlled process during which larger patterns emerge driving the location of the final necks. At high stretching rates, it seems that the second effect is enhanced with, accordingly, a pattern size almost insensitive to the grain size and a limited variability with the grain structure occurrence.
\end{abstract}

\section{Introduction}

The localization of plastic deformation in metal plates under dynamic stretching in the form of necking, which leads to the fragmentation of the structure, is a well-documented topic. Its initiation is usually addressed at the macroscopic scale as an outcome of the development of unstable perturbation modes of the homogeneous expansion. Linear stability analysis approaches for different geometries and behaviors have been developed following the early papers of Fressengeas and Molinari [1] and Shenoy and Freund [2]; let us mention for instance [3], [4] or [5] among the numerous works on this topic. On the other hand, the late stages are assumed to be governed by a defect interaction process during which stress release waves are emitted from the points in which necking is the most pronounced and inhibit the growth of other necks in the area they sweep: the starting point of this kind of analysis was proposed by Mott [6]. Yet, at the mesoscale, i.e. the one of the crystalline structure, the deformation is inherently heterogeneous since the beginning of loading. The connection between the initial strain distribution, closely linked with the grain structure, and the latest localization pattern needs to be investigated. In the following, such

\footnotetext{
*Corresponding author: jean-lin.dequiedt@cea.fr
} 
an analysis is developed for a tantalum sheet submitted to plane strain stretching (more details are provided in [7]). The crystal plasticity model and simulated geometry are presented in Section 2. The different steps of the localization process are displayed in Section 3 for two numbers of grains and the sensitivity to the local behavior is discussed. The influence of stretching rate is analyzed in Section 4 and the variability of results with the set of lattice orientations is evaluated in Section 5.

\section{Crystal plasticity model and simulated geometry}

The single crystal constitutive behavior is formulated in a finite deformation framework and plasticity is ruled by a Teodosiu type model taking dislocation densities as internal state variables [8]; the deformation gradient tensor is decomposed into an elastic and a plastic part:

$$
\mathbf{F}=\mathbf{F}^{e} \cdot \mathbf{F}^{p}
$$

The elastic transformation $\mathbf{F}^{e}$ encompasses all lattice deformations and rotations (the relaxed configuration is said "isoclinic") and the plastic transformation rate expresses as a sum of slip rates on the different slip systems of the crystal lattice:

$$
\mathbf{L}^{p}=\dot{\mathbf{F}}^{p} \cdot \mathbf{F}^{p-1}=\sum_{\alpha} \dot{\gamma}^{\alpha} \mathbf{m}_{0}^{\alpha} \otimes \mathbf{n}_{0}^{\alpha} .
$$

In the case of BCC structures such as tantalum, there are 24 slip systems, 12 with $\{110\}$ slip planes and 12 with $\{112\}$ slip planes. Slip rates are governed by a thermal activation law:

$$
\dot{\gamma}^{\alpha}=\dot{\gamma}_{0} \exp \left(-\frac{\Delta G}{k_{B} T}\right) \quad \text { with } \quad \Delta G=\Delta F\left(1-\left(\frac{\tau^{\alpha}-\tau_{c}^{\alpha}}{s}\right)^{p}\right)^{q} .
$$

The resolved shear stress $\tau^{\alpha}$ is the projection of the stress tensor on the slip system and expresses in finite deformations ( $\pi$ is the second Piola-Kirchhoff stress tensor related to the relaxed configuration):

$$
\tau^{\alpha}=\left(\mathbf{F}^{e T} \cdot \mathbf{F}^{e} \cdot \boldsymbol{\pi}\right):\left(\mathbf{m}_{0}^{\alpha} \otimes \mathbf{n}_{0}^{\alpha}\right)
$$

The more or less favorable orientation of one system towards the loading state is given by the Schmid factor which can be defined by extending the uniaxial stress formulation, $\|\mathbf{A}\|$ being the "von Mises norm" of tensor A:

$$
\eta^{\alpha}=\frac{\left|\tau^{\alpha}\right|}{\left\|\mathbf{F}^{e T} \cdot \mathbf{F}^{e} \cdot \mathbf{\pi}\right\|} \text { with }\|\mathbf{A}\|=\sqrt{\frac{3}{2} \mathbf{A}^{d}: \mathbf{A}^{d}} \text { and } \mathbf{A}^{d}=\mathbf{A}-\frac{1}{3} \operatorname{tr}(\mathbf{A}) \mathbf{I} .
$$

Strain hardening is quantified by the evolution of the critical shear stress with dislocation densities per system:

$$
\tau_{c}^{\alpha}=\tau_{0}+\mu b \sqrt{\sum_{\beta} a^{\alpha \beta} \rho^{\beta}}
$$


The hardening matrix $a^{\alpha \beta}$ is derived from dislocation dynamics simulations and coefficients for tantalum are given in [9]. The evolution of dislocation densities is driven by the competition between dislocation multiplication and annihilation by dynamic recovery:

$$
\dot{\rho}^{\alpha}=\frac{1}{b}\left(\sqrt{\sum_{\beta} d^{\alpha \beta} \rho^{\beta}}-2 y_{c} \rho^{\alpha}\right) \dot{\gamma}^{\alpha} .
$$

The maximum Schmid factor when all slip systems are accounted for $\left(\eta^{\max }=\right.$ $\max _{\alpha}\left(\eta^{\alpha}\right)$ ) is a measure of the local resistance to slip in the absence of strain hardening: when all critical shear stresses are equal, slip is favored by a high $\eta^{\max }$.

Sheet simulations (cf. Fig. 1) are performed with the crystal plasticity code Coddex using an Element Free Galerkin numerical scheme.

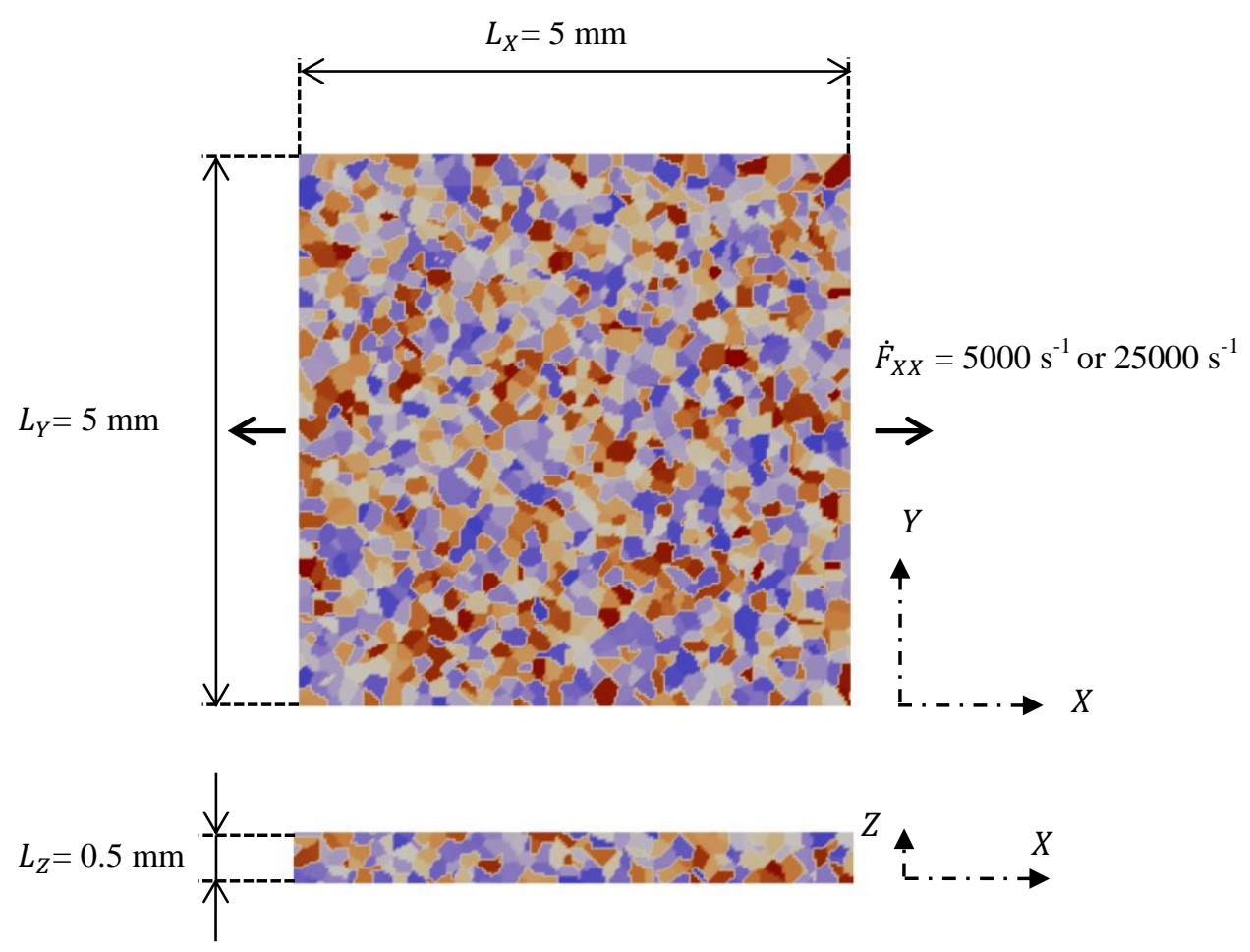

Fig. 1. Simulated stretching sheet in the case of a 4000 grain aggregate.

A polycrystalline structure is generated by a Voronoï tessellation algorithm and both 4000 and 50000 grain aggregates are considered: a random selection of 100 crystal orientations is distributed among these grains. Periodic boundary conditions and an average plane strain deformation gradient $\mathbf{F}\left(F_{Y Y}=0\right)$ are imposed in the sheet plane, the upper and lower faces being free of loading: the two stretching rates $\dot{F}_{X X}=5000 \mathrm{~s}^{-1}$ and $25000 \mathrm{~s}^{-1}$ are considered. A mesh of $200 * 200 * 20$ nodes is chosen as a compromise between computational cost and resolution (for the 50000 grain structure, it amounts to an average of 16 nodes per grain). 


\section{Development of necking in plane strain stretching}

\subsection{Main features for small and large number of grains}

Computation with stretching rate $\dot{F}_{X X}=5000 \mathrm{~s}^{-1}$ is presented in this subsection. The development of necking is investigated by plotting maps of Hencky strain $\varepsilon^{\text {dev }}$ at different times of the loading process. Namely:

$$
\varepsilon^{d e v}=\sqrt{\frac{2}{3} \boldsymbol{\varepsilon}^{d}: \boldsymbol{\varepsilon}^{d}} \text { with } \boldsymbol{\varepsilon}^{d}=\boldsymbol{\varepsilon}-\frac{1}{3} \operatorname{tr}(\boldsymbol{\varepsilon}) \mathbf{I} \text { and } \boldsymbol{\varepsilon}=\frac{1}{2} \log \left(\mathbf{F}^{T} \cdot \mathbf{F}\right)
$$

The cases corresponding to the two numbers of grains are displayed on Fig. 2.
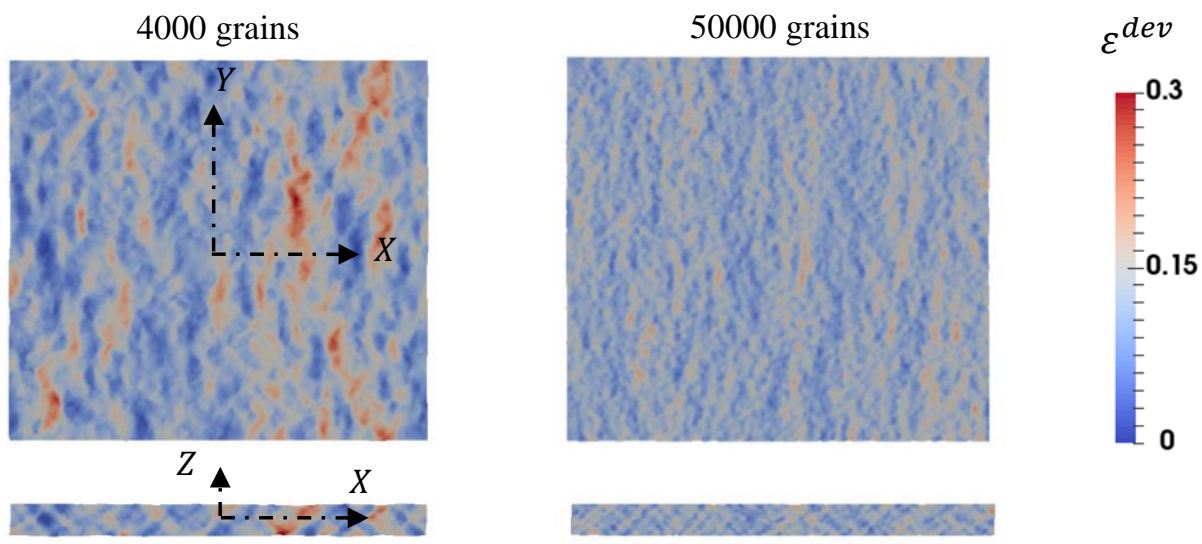

$$
t=20 \mu \mathrm{s} \text { and } F_{X X}=1.1
$$

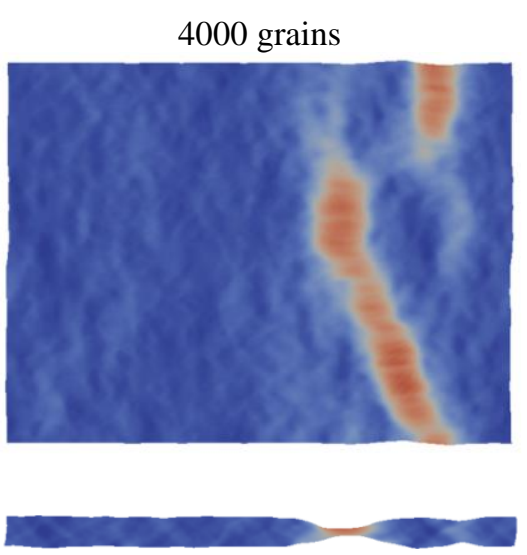

$t=65 \mu$ s and $F_{X X}=1.325$

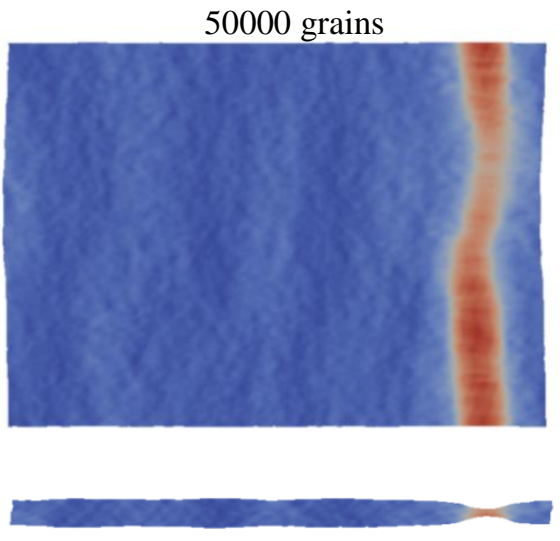

$t=80 \mu \mathrm{s}$ and $F_{X X}=1.4$ $\varepsilon^{d e v}$

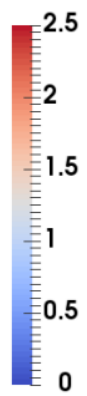

Fig. 2. Maps of deviatoric (Hencky) strain $\varepsilon^{d e v}$ in average plane and cross section for the 4000 and 50000 grain aggregates for stretching rate $\dot{F}_{X X}=5000 \mathrm{~s}^{-1}$. 
In the sheet plane, a bidimensional strain organization with fluctuations at the scale of a few grains is displayed in the early stage which evolves towards the formation of patterns with a prevailing orientation in the $Y$ direction. In the late stage, only one localization zone is visible.

For the 4000 grain structure, it clearly takes a path along which the strain was initially high. For the 50000 grain structure, memory of the initial strain pattern is less clear and the localization zone is almost aligned with the $Y$-axis, as would be predicted by a macroscopic necking criterion. In the cross section, an initial strain organization approximately oriented $45^{\circ}$ from the sheet plane triggers the formation of one main neck developing up to cross section collapse (failure is not modeled in these calculations). The localization process is delayed when the grain number increases consistently with lower initial plastic strain heterogeneity in this case.

The correlation between the strain pattern and intragranular slip behavior at the beginning of loading is evaluated by plotting strain profiles along the $X$-axis superimposed with maximum Schmid factor profiles. For the 4000 grain aggregate, a partial but not systematic correlation is exhibited between high deviatoric strain and high Schmid factor (Fig. 3): in other words, strain first concentrates in the grains which offer the lowest resistance due to the orientation of their slip systems, the effect of strain hardening being of second order at this short time. This feature is less clear for the 50000 grain aggregate: due to the higher number of grains in the sheet thickness, the deformation in one grain is more influenced by surrounding grains.

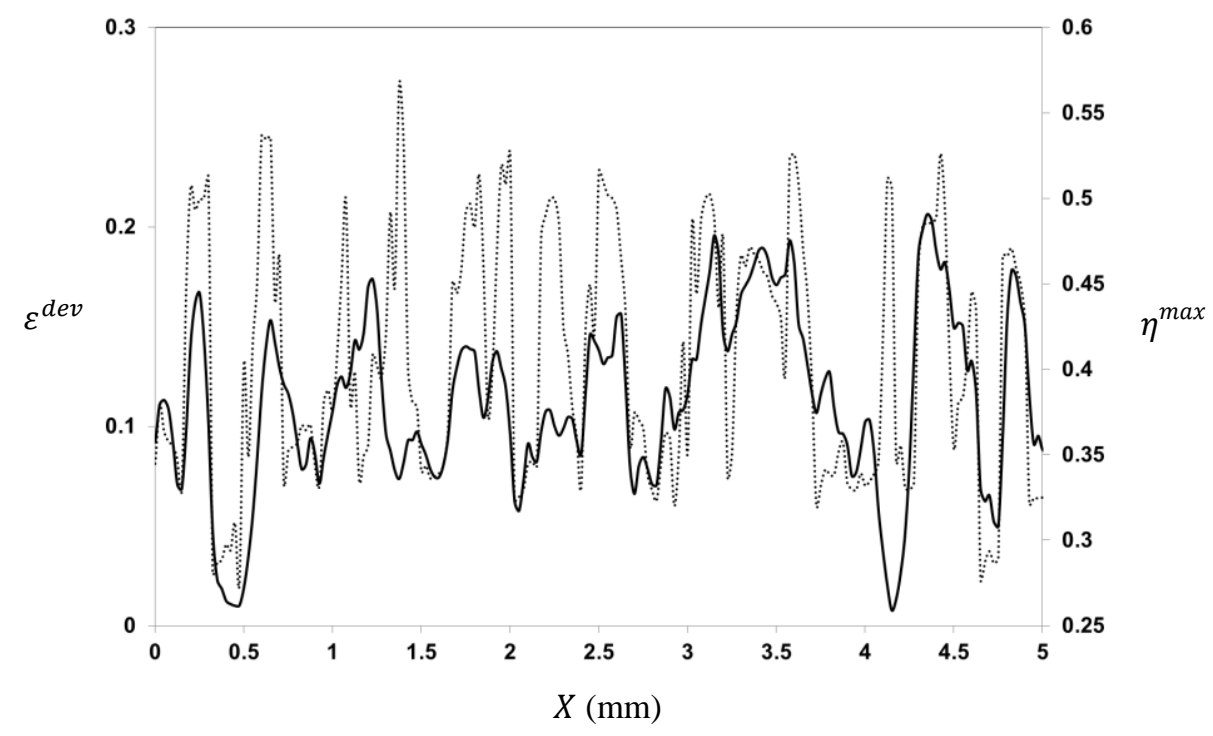

Fig. 3. Strain (solid line) and Schmid factor (dotted line) profiles on the $X$-axis for the 4000 grain aggregate for stretching rate $\dot{F}_{X X}=5000 \mathrm{~s}^{-1}$ at time $t=20 \mu \mathrm{s}$. 


\subsection{Influence of stretching rate}

When the stretching rate is raised to $25000 \mathrm{~s}^{-1}$, three necking bands remain up to the late stage one of them being located at the same place as the one identified at $\dot{F}_{X X}=5000 \mathrm{~s}^{-1}$ (see top of Fig. 4 for the reference 50000 grain microstructure). Moreover, strong localization occurs for higher elongation than for $\dot{F}_{X X}=5000 \mathrm{~s}^{-1}$ and is preceded by an intermediate stage during which a modulation with a wavelength of the order of the sheet thickness develops.

\section{Reference 50000 grain microstructure}

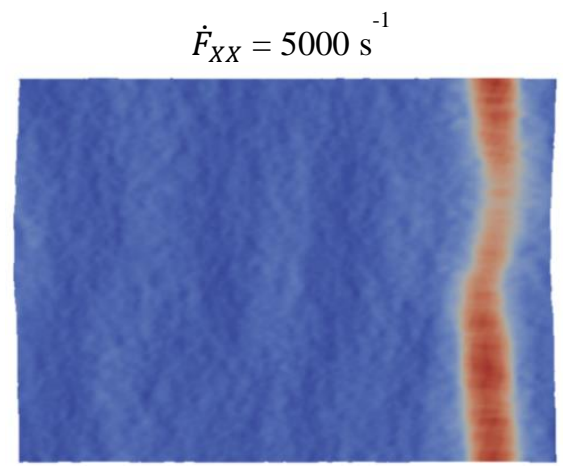

$t=80 \mu \mathrm{s}$ and $F_{X X}=1.4$

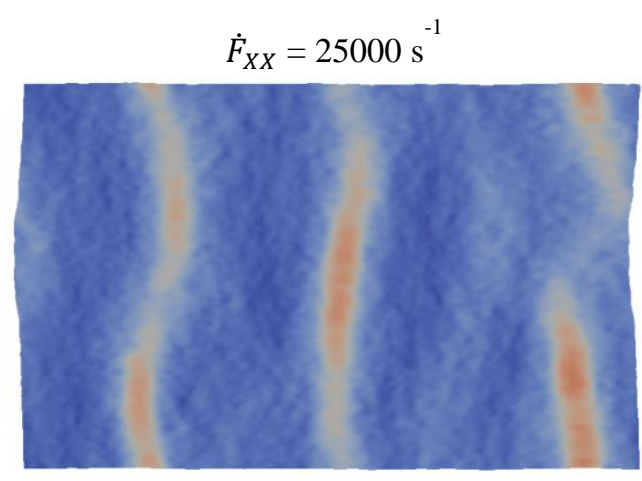

$t=24 \mu \mathrm{s}$ and $F_{X X}=1.6$

\section{Second set of crystal orientations}

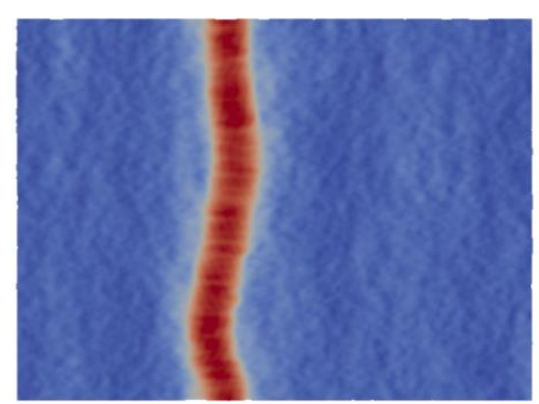

$t=70 \mu \mathrm{s}$ and $F_{X X}=1.35$

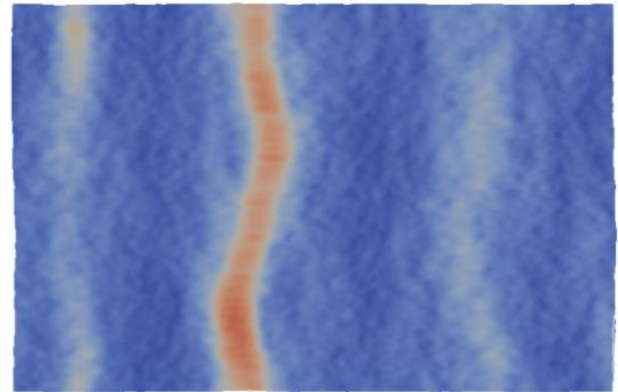

$t=22 \mu \mathrm{s}$ and $F_{X X}=1.55$

Fig. 4. Maps of Hencky strain $\varepsilon^{d e v}$ in average plane for the 50000 grain aggregate and plane strain stretching in the late stage for stretching rates $\dot{F}_{X X}=5000 \mathrm{~s}^{-1}$ and $\dot{F}_{X X}=25000 \mathrm{~s}^{-1}$ (same color scale as Fig. 2). Computation with a second set of crystal orientations is also reported (see Section 3.3)

A deeper analysis of the transition from a "weakest link" driven process to a one in which sheet instability modes have increasing influence is provided by representing the strain profiles at different times for the 50000 grain structure and the two stretching rates (Fig. 5). 

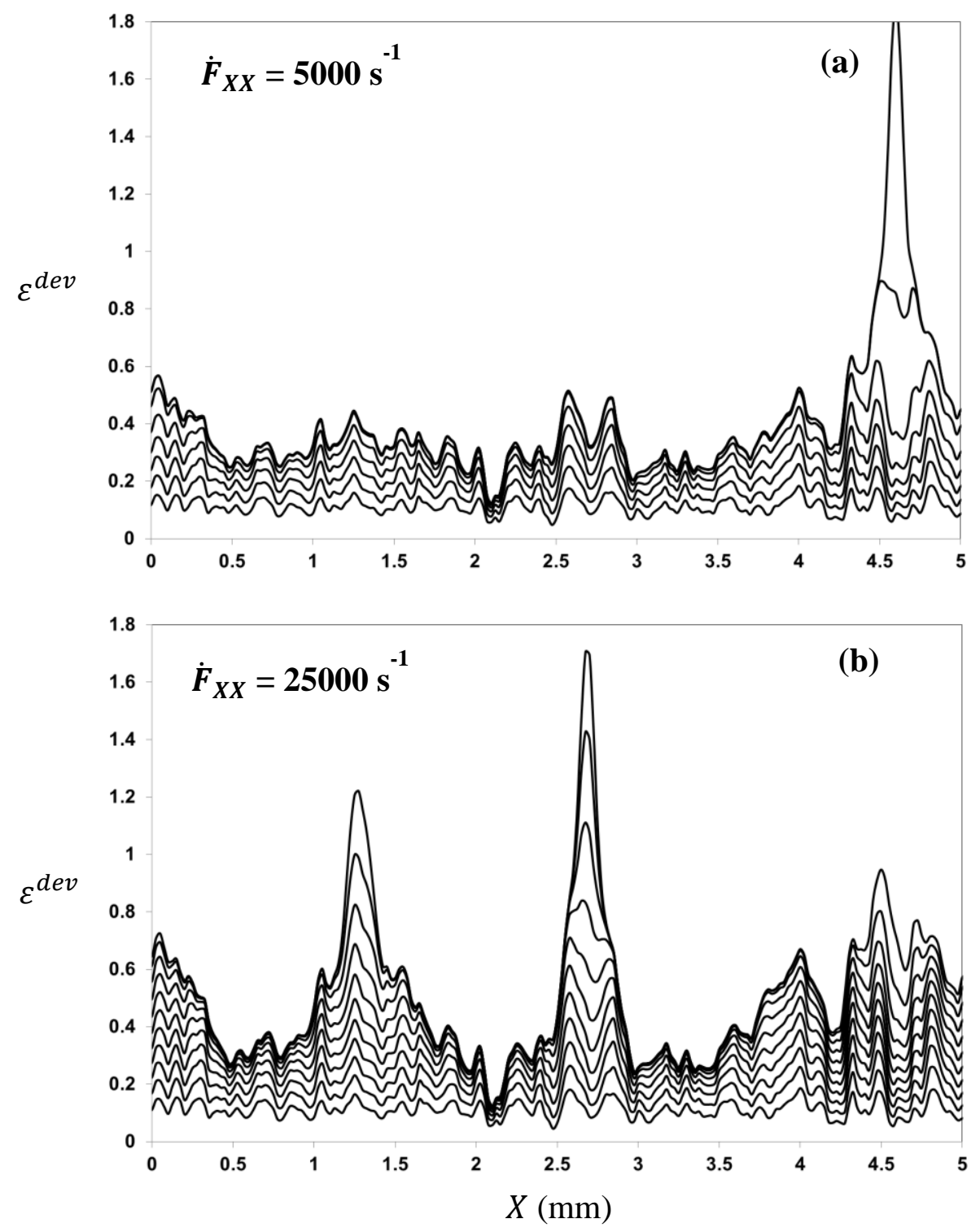

Fig. 5. Strain profile for the 50000 grain aggregate on the $X$-axis (a) at times $t=20 \mu \mathrm{s}, 40 \mu \mathrm{s}, 60 \mu \mathrm{s}$, $80 \mu \mathrm{s}$ and $90 \mu \mathrm{s}$ for stretching rate $\dot{F}_{X X}=5000 \mathrm{~s}^{-1}$ and (a) at times $t=4 \mu \mathrm{s}, 8 \mu \mathrm{s}, 12 \mu \mathrm{s}, 16 \mu \mathrm{s}, 20 \mu \mathrm{s}$ and $24 \mu$ s for stretching rate $\dot{F}_{X X}=25000 \mathrm{~s}^{-1}$.

For moderate stretching rate $\left(\dot{F}_{X X}=5000 \mathrm{~s}^{-1}\right)$, the initial strain profile, linked with the microstructure, first grows uniformly keeping its shape and a slight long wavelength modulation appears. Next, one peak covering about two maxima of this profile grows up very quickly leading to the necking band observed on Fig. 4; it inhibits deformation in the rest of the sheet as could be predicted by a Mott type obscuration approach. For the highest stretching rate, the modulation has much more time to develop and several peaks form at the locations of its maxima; everything happens as if inertia shifted strong localization to 
higher elongation and strengthened the influence of structural modes. A comparison with the 4000 grain aggregate at the same stretching rate, not reported here, seems to prove that the modulation wavelength is not controlled by the grain size, which enforces the assumption of a macroscopic instability mode. At last, let us mention that, when the stretching rate increases, the Mott obscuration waves are expected to propagate on shorter distances (see [10] for instance).

\subsection{Variability with microstructure occurrence}

The variability of results with the microstructure is evaluated by computing, for the same set of grain shapes (same set of Voronoï seeds), a second instance of grain lattice orientations. The strain maps obtained for the 50000 grain microstructure display, for the two stretching rates, the same number of necking bands between the two occurrences of lattice orientations albeit located in different places (bottom of Fig. 4). The variability of elongation $F_{X X}$ at necking time is limited and seems to be lower when the stretching rate increases which could be interpreted as an outcome of the influence of inertia on the necking process at high stretching rates.

\section{Conclusion}

The incidence of microstructural heterogeneity on the development of plastic strain localization such as necking and the interplay with instability modes as predicted by macroscopic approaches is a challenging topic. It can be addressed thanks to polycrystal plasticity simulations, even though a refined grain meshing is hardly compatible with reasonable computation times. The strong anisotropy of single crystal plastic behavior yields heterogeneous deformation fields since the very beginning of loading. In stretching sheets, when the number of grains in the thickness is low and the loading rate is moderate, the most favorably oriented grains offer less resistance to deformation and act as a distribution of point defects interacting by an obscuration process; they drive the location of necking zones. On the contrary, when the grain number increases, the microstructure heterogeneity once averaged in the thickness is somehow smoothed and the "weak link effect" is reduced. Moreover, at high loading rates, strong localization is delayed by inertia and leaves more time for structural perturbation modes to develop.

At last, this kind of approach opens the way to study the influence of microstructural characteristics inherited from the sheet forming process on the development of plastic strain localization: a marked texture, non-isotropic grain shapes or heterogeneity of initial dislocation densities (which could be identified in partly recrystallized materials for instance) could perfectly be incorporated in the computation.

\section{References}

1. C. Fressengeas, A. Molinari, Eur. J. Mech. A/ Solids, 13, 251 (1994)

2. V.B. Shenoy, L.B. Freund, J. Mech. Phys. Solids, 47, 2209 (1999)

3. S. Mercier, A. Molinari, Int. J. Solids Struct., 40, 1995 (2003) 
4. J.A. Rodriguez-Martinez, A. Molinari, R. Zaera, G. Vadillo, J. Fernandez-Saez, Int. J. Solids Struct., 108, 74 (2017)

5. M. Xavier, C. Czarnota, D. Jouve, S. Mercier, J.L. Dequiedt, A. Molinari, Eur. J. Mech. A/ Solids, 79, 1 (2020)

6. N.F. Mott, In: Proc. Roy. Soc., Series A, 300 (1947)

7. J.L. Dequiedt, C. Denoual, Int. J. Solids Struct., 210-211, 183 (2021)

8. C. Teodosiu, J.L. Raphanel, L. Tabourot, Finite element simulation of the large elastoplastic deformation. In: Large Plastic Deformations, Rotterdam, A.A. Balkema, 153 (1991)

9. R. Madec, L.P. Kubin, Acta Mater., 126, 166 (2017)

10. C. Denoual, F. Hild, Comp. Meth. Appl. Mech. Eng., 183(3), 247 (2000) 